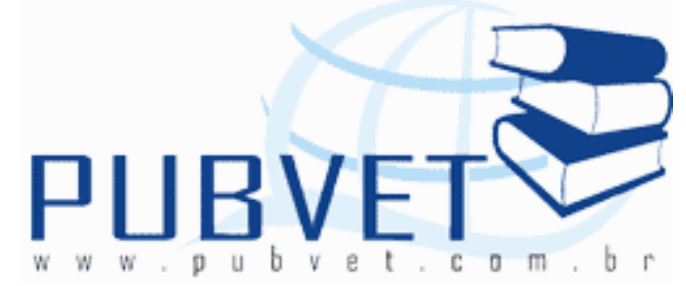

PUBVET, Publicações em Medicina Veterinária e Zootecnia.

\title{
Implantes poliméricos intraoculares de fármacos na medicina veterinária - revisão de literatura e relato de um caso
}

Flávia Biondi ${ }^{1}$, Fabiano Montiani-Ferreira ${ }^{2}$, Gustavo de Oliveira Fulgêncio ${ }^{3}$, Karen Medina Teixeira ${ }^{4}$, Peterson Triches Dornbush ${ }^{5}$, Armando da Silva Cunha Junior $^{6}$

${ }^{1}$ Médica Veterinária, mestranda do Programa de Pós Graduação em Ciências Veterinárias da Universidade Federal do Paraná. Rua dos Funcionários, 1540, CEP 80 035-050 - Cabral - Curitiba - Paraná - Brasil. Email: flabiondi@hotmail.com

${ }^{2}$ Médico Veterinário, Professor Adjunto IV de Clínica Médica de Pequenos Animais e Oftalmologia Veterinária da Universidade Federal do Paraná.

${ }^{3}$ Médico Veterinário, doutorando da Universidade Federal de Minas Gerais.

${ }^{4}$ Médica Veterinária, residente de Oftalmologia Veterinária da Universidade Federal do Paraná.

${ }^{5}$ Médico Veterinário, Professor Adjunto de Clínica Cirúrgica de Pequenos Animais e Clínica Cirúrgica de Grandes Animais da Universidade Federal do Paraná.

${ }^{6}$ Farmacêutico, Professor associado de farmácia da Universidade Federal de Minas Gerais.

\section{Resumo}

Doenças do segmento posterior do bulbo do olho são responsáveis pela maioria dos casos de cegueira irreversível no mundo inteiro e, até o momento, 
apresentam sucesso terapêutico limitado devido à dificuldade de penetração dos fármacos neste local. Na medicina veterinária, as doenças oculares crônicas apresentam frequente insucesso terapêutico por conta da frequência e via de administração, além do alto custo das medicações. Visando a obtenção de níveis terapêuticos adequados de fármacos por períodos prolongados e a diminuição de efeitos colaterais indesejados, alguns sistemas de liberação poliméricos de implantação intraocular estão sendo investigados. Eles são preparados na forma de implantes a partir de polímeros que podem ser biodegradáveis ou não biodegradáveis, e pretendem revolucionar a oftalmologia humana e veterinária, aumentando o conforto do paciente e a eficácia medicamentosa no tratamento de doenças até o momento tidas como irreversíveis por conta das dificuldades terapêuticas á elas relacionadas.

Palavras-chave: doenças oculares; medicação intraocular; sistemas de liberação.

\title{
Polymeric implants for intraocular administration of drugs in veterinary medicine - review of the literature and report of a case
}

\begin{abstract}
Diseases of the posterior segment of the eye are responsible for most cases of irreversible blindness, and until at the present moment, have limited therapeutic success due to the difficulty of penetration of drugs in this site. In veterinary medicine, chronic eye diseases are often frustrating to treat due to the frequency and route of administration, besides the high cost of the drugs involved. In order to obtain therapeutic levels of these drugs for prolonged periods and to promote reduction of unwanted side effects, some polymeric delivery systems designed for intraocular implantation are currently being investigated. They are prepared in the form of implants from polymers that can be classified in biodegradable or nonbiodegradable, and are aimed to revolutionize medical and veterinary ophthalmology, increasing patient comfort
\end{abstract}



2012.

and effectiveness of drugs in the treatment of diseases, which are so far regarded as irreversible because of the difficulties therapies related to them.

Keywords: eye diseases; intraocular medication; systems release.

\section{INTRODUÇÃO}

As doenças oculares acometem as mais diversas espécies, incluindo a espécie humana, podendo causar intenso desconforto, comprometimento da acuidade visual e, por fim, cegueira. Sabe-se que as doenças do segmento posterior do olho são responsáveis pela maioria dos casos de cegueira irreversível no mundo (Fialho e Cunha Júnior, 2007). Em seres humanos, crescem os casos de catarata, retinopatia diabética, glaucoma, degeneração macular, uveíte crônica, entre outros exemplos. Para todos esses casos, o emprego de medicações de uso contínuo geralmente são necessários e o sucesso no tratamento destas doenças visa, essencialmente, o transporte de doses efetivas de fármacos para os tecidos mais internos do olho.

A estrutura anatômica e fisiológica do olho faz com que o alcance de fármacos no seu segmento posterior seja imprevisível. As soluções oftálmicas para administração tópica possuem pouco ou nenhum efeito terapêutico nessas estruturas e ainda podem sofrer alterações dependentes da produção lacrimal, impermeabilidade do epitélio corneano e tempo de permanência transitório no local de aplicação. As injeções intraoculares de fármacos são utilizadas por alcançarem altos níveis de eficácia, no entanto, múltiplas injeções estão associadas a um maior risco de catarata, hemorragias vítreas, descolamento de retina e endoftalmites, sem contar ainda o desconforto causado para o paciente (Choonara et al., 2011). Os fármacos administradas pela via sistêmica atingem o segmento posterior do olho através da circulação sanguínea, e devido à complexa estrutura de defesa compreendida pelas barreiras intraoculares, a utilização desta via na terapêutica ocular requer, geralmente, a administração de doses elevadas visando a manutenção de níveis terapêuticos adequados por períodos prolongados, o que pode acarretar 
em severos efeitos adversos (Fialho e Cunha Júnior, 2007). Conclue-se, dessa forma, que a baixa penetração de fármacos no interior do olho limita o número de medicações indicadas para o uso em oftalmologia, e exige cuidados com aquelas disponíveis devido a possíveis ocorrências de efeitos adversos. A administração de fármacos para o tratamento de doenças oculares deve ser feita, de preferência, por meio de vias que atinjam o tecido local, visando minimizar a ocorrência de efeitos adversos e a absorção sistêmica.

Na medicina veterinária, há maiores dificuldades no que corresponde ao tratamento de animais com doenças oculares crônicas. A aplicação constante de medicações é dificultada pelo temperamento de alguns animais domésticos e a natureza dos animais selvagens, tornando-se praticamente impossível neste caso. Ainda, o alto custo das medicações e a frequência das administrações acaba por inibir a maioria dos proprietários, que não conseguem arcar com o tratamento durante toda a vida do seu animal.

Devido a todas as dificuldades encontradas, algumas pesquisas têm sido realizadas no sentido de desenvolver sistemas de administração intraoculares que permitam liberar concentrações terapêuticas dos fármacos por um período prolongado e adequado (Rubsamen et al., 1994; Hashizoe et al., 1994; Chang e Wong, 1999; Gilger et al., 2000; Avitabile et al., 2001; Okabe et al., 2003; Sakurai et al., 2003; Saliba et al., 2008; Choonara et al., 2011; Haghjou et al., 2011). Tais sistemas podem proporcionar inúmeras vantagens, como aumentar a biodisponibilidade do fármaco, no sentido de obter uma liberação constante e prolongada; aumentar as concentrações locais, sem a ocorrência de efeitos adversos sistêmicos; atingir mais especificamente um tipo de tecido ou célula; reduzir a freqüência de injeções intraoculares. Tais vantagens podem aumentar o conforto do paciente e reduzir as complicações observadas das injeções intraoculares (Behar-Cohen, 2002).

Este trabalho tem como objetivo revisar a necessidade e propriedades de materiais sintéticos ou biodegradáveis para a aplicação em oftalmologia 
veterinária, bem como relatar um caso de aplicação intravítrea de um polímero biodegradável em um animal da espécie equina.

\section{DESENVOLVIMENTO}

O desenvolvimento de novos sistemas de distribuição de drogas direcionadas ao segmento posterior do olho se torna necessário, e pode oferecer inúmeras vantagens, tais como o aumento de biodisponibilidade do fármaco e a liberação do mesmo de forma local e constante sem efeitos colaterais sistêmicos, aumentando o conforto do paciente (Fialho et al., 2006).

Os implantes são preparados a partir de diferentes polímeros, sendo eles biodegradáveis ou não-biodegradáveis. Os polímeros biodegradáveis são aqueles que apresentam uma redução da massa molar quando em contato com o ambiente corpóreo. A degradação pode ser vista como um conjunto de processos, estimulados por agentes externos, que levam a modificações da estrutura dos polímeros, as quais trarão alterações também nas propriedades do material. As degradações podem ser originadas tanto da atuação de entidades biológicas como de células, microorganismos, enzimas, como do ataque de espécies iônicas, radicais livres ou água. Em meio biológico, as formas mais comuns de degradação são a hidrólise e a degradação por enzimas. A degradação por enzimas pode ser altamente variável de pessoa para pessoa, pois os níveis enzimáticos podem diferir bastante. Os polímeros biodegradáveis têm sido extensivamente pesquisados e utilizados em materiais, visto que não precisam ser removidos e não causam indesejáveis efeitos em longo prazo. Existem várias aplicações para estes tipos de biopolímeros: suturas, dispositivos para liberação de fármacos, fixação de dispositivos ortopédicos, vasos sanguíneos temporários e matriz para engenharia de tecidos. Eles devem ser um meio adequado para o crescimento dos tecidos, guiando a resposta tissular, favorecendo a fixação e proliferação celular e controlando a resposta inflamatória e imunológica (Bispo, 2009). 
Sistemas não biodegradáveis necessitam de remoção após completa liberação do fármaco (Fialho e Cunha Júnior, 2007; Choonara et al., 2011)

Estes sistemas podem, ainda, ser de dois tipos: matriciais (ou monolíticos) e reservatórios (Dash e Cudworth II, 1998; Kimura e Ogura, 2001). No sistema matricial, a substância se encontra dispersa na matriz polimérica. No sistema do tipo reservatório, preparados com polímeros biodegradáveis ou não biodegradáveis, a substância se encontra em uma cavidade central envolta por uma membrana polimérica, a qual controla sua taxa de liberação. Quando se utilizam polímeros não biodegradáveis, a liberação ocorre apenas por um processo de difusão lenta pela matriz. (Dash e Cudworth II, 1998; Kimura e Ogura, 2001).

A liberação do fármaco, caso se trate de um sistema biodegradável, ocorre por difusão pelos poros da matriz, por degradação do polímero ou por uma combinação dos dois mecanismos. Dessa forma, os implantes intraoculares preparados com polímeros biodegradáveis não precisam ser posteriormente removidos, pois são completamente metabolizados e eliminados pelo organismo. Polímeros biodegradáveis naturais e sintéticos estão sendo estudados como componentes em sistemas de liberação de fármacos, mas nem todos têm demonstrado verdadeira biocompatibilidade. Mesmo assim polímeros naturais tem ganhado grande destaque em pesquisas recentes. O colágeno, por exemplo, depois de purificado é usado em implantes cardiovasculares e como matriz para crescimento de tecidos. A celulose modificada é utilizada na imobilização e desenvolvimento de células e a quitosana também vem sendo utilizada para matrizes de engenharia de tecido. Isto se dá porque muitos destes polímeros naturais têm conseguido aprovação do FDA (Bispo, 2009). Já os sintéticos, representados por poliamidas, poliaminoácidos, polialquilcianacrilatos, poliésteres, poliortoésteres, poliuretanos e poliacrilamidas, têm apresentado crescente interesse na aplicação como sistemas de liberação de fármacos. Os polímeros biodegradáveis mais utilizados atualmente são os poliésteres, tais como a poli 
(e-caprolactona), o PLA (poli (ácido lático)) e os diferentes tipos de PLGA (poli ( $D$, L lático-co-glicólico)), sendo que os dois últimos tipos têm sido amplamente empregados (Jain et al., 1998).

Alguns implantes não biodegradáveis de liberação lenta foram aprovados para uso clínico nos Estados Unidos da América (EUA): o Ocusert $®$ (Alza, Mountain View, California, EUA), um dispositivo conjuntival que libera pilocarpina, o Vitrasert $\AA$ (Bausch \& Lomb, Rochester, NY, EUA), um implante intravítreo contendo ganciclovir, e o Retisert ${ }^{\circledR}$ (Bausch \& Lomb, Rochester, NY, EUA), um implante intravítreo contendo fluocinolona indicado para o tratamento de uveíte não infecciosa crônica. Outros sistemas compostos por polímeros não biodegradáveis incluem os derivados de celulose, silicones, polímeros acrílicos, polivinilpirrolidona e copolímeros dos óxidos de etileno e propileno, que apresentam uma taxa de liberação relativamente constante, porém também precisam ser removidos posteriormente, o que requer novos processos cirúrgicos.

Os implantes preparados a partir de sistemas poliméricos podem ser aplicados em diferentes regiões do olho. Ordenando-se as regiões da mais superficial a mais profunda, temos: região subconjuntival, região subtenoniana, esclera e o interior do bulbo do olho (câmara anterior e corpo vítreo). Em geral quanto mais profunda a região, mais delicado o procedimento e mais eficaz a concentração no vítreo e na retina (Kimura e Ogura, 2001; Fialho et al., 2003). Os implantes intravítreos evitam o problema de redução da transparência do meio ocular e têm sido investigados para o tratamento de diferentes doenças intraoculares e também para aplicação em cirurgias de catarata.

Os polímeros biodegradáveis têm sido largamente utilizados no campo médico e farmacológico como sistemas de distribuição de fármacos (Saliba et al., 2008), e vários trabalhos têm sido realizados para avaliar a eficácia destes como implantes intraoculares. Hashizoe e colaboradores desenvolveram um 
implante biodegradável contendo doxorrubicina para o tratamento de vitreorretinopatia proliferativa experimental em coelhos e a taxa de liberação do fármaco no corpo vítreo foi avaliada (Hashizoe et al., 1994). Esse sistema, composto pelo polímero poli ( $D, L$ lático-co-glicólico) (PLGA) e contendo $1 \%$ de doxorrubicina, foi implantado na pars plana, dessa forma não alterando a transparência do meio ocular, pois foi capaz de promover a liberação diretamente no corpo vítreo. Os estudos in vitro realizados mostraram que $26 \%$ do fármaco foram liberados durante quatro semanas, e os in vivo mostraram uma manutenção da concentração da doxorrubicina no corpo vítreo dentro da faixa terapêutica por um período superior a quatro semanas. Não foi observada nenhuma reação tóxica significativa. As avaliações foram feitas por eletrorretinografia e histopatologia do local.

Foi estudada igualmente, a eficácia de um implante à base de PLGA, contendo 5-fluoruracila (5-FU), para o tratamento de vitreoretinopatia proliferativa (PVR) (Rubsamen et al., 1994). As concentrações intravítreas do fármaco foram mantidas entre 1,0 e $13,0 \mu \mathrm{g} / \mathrm{mL}$ por 14 dias, e permaneceram acima de $0,3 \mu \mathrm{g} / \mathrm{mL}$ por aproximadamente 21 dias. A implantação do sistema se mostrou eficaz na inibição de $P V R$, e não demonstrou sinais de efeitos tóxicos na retina.

A biocompatibilidade e a biodegradação in vivo de implantes intravítreos preparados com diferentes ésteres de ácido hialurônico foi avaliada em olhos de coelhos (Avitabile et al., 2001). Os sistemas preparados com os derivados etil éster e benzil éster do ácido hialurônico na concentração de $100 \%$ foram degradados em 60 e 150 dias, respectivamente. O implante à base de benzil éster a $75 \%$ foi completamente reabsorvido após 15 dias. Estudos de microscopia, oftalmoscopia e eletrorretinografia realizados mostraram que os implantes preparados com ácido hialurônico são biocompátiveis e não foram observados sinais de inflamação ocular. 

2012.

A segurança e a eficácia de um sistema biodegradável composto de PLGA e contendo $0,5 \mathrm{mg}$ de ciclosporina (CSA) foram avaliadas após sua implantação na câmara anterior de olhos de coelhos (Theng et al., 2003). Foram encontradas concentrações elevadas do fármaco nas camadas da córnea (epitélio anterior, substância própria e epitélio posterior) durante os 3 meses do estudo. Baixas concentrações de CSA foram detectadas no humor aquoso e não foi detectado o fármaco no sangue. Reações adversas não foram observadas durante o estudo.

Um sistema biodegradável à base de PLA e contendo fosfato de betametasona foi implantado na esclera de olhos de coelhos (Okabe et al., 2003). Foram realizados exames de oftalmoscopia, eletrorretinografia e microscopia óptica para avaliar a toxicidade e a biocompatibilidade dos implantes. Os resultados in vitro mostraram que o fármaco foi liberado por 8 semanas. A concentração de betametasona foi mantida dentro da faixa terapêutica no vitreo e na retina-coróide por mais de 8 semanas. Não foi observada reação tóxica na retina durante o estudo e o implante apresentouse biocompatível com o tecido ocular.

A eficácia de um implante polimérico biodegradável contendo tacrolimus foi avaliada em um modelo animal de uveíte experimental (Sakurai et al., 2003). Os sistemas desenvolvidos foram implantados no corpo vítreo dos olhos de coelhos e o grau de inflamação na câmara anterior e no corpo vítreo foram avaliados através de análises histológicas. A função retiniana também foi avaliada. As análises histopatológicas mostraram que os olhos tratados apresentaram menores sinais de inflamação, enquanto os olhos não tratados apresentaram marcante inflamação e grande desorganização tecidual. A liberação controlada de tacrolimus a partir destes implantes foi efetiva na supressão da inflamação de uveíte experimental por 6 semanas.

A uveíte recorrente equina (URE), também conhecida como cegueira da lua, iridociclite e oftalmia periódica é a causa mais comum de cegueira em 
equinos, e representa a doença mais devastadora para a indústria equina em todo o mundo (Keller e Hendrix, 2005). A natureza progressiva da URE é responsável pelo subsequente desenvolvimento de catarata, adesões intraoculares, phthisis bulbi e cegueira, e em associação com as dificuldades encontradas no tratamento são as maiores causas de eutanásia em cavalos, realizadas por segurança ou razões éticas e humanitárias (Deeg et al., 2001; Gilger et al., 1999). Acredita-se que a URE tenha uma importante parcela imunomediada na sua patogênese (Deeg et al., 2001), e tem sido estudada com intensidade pela semelhança com as uveítes recorrentes em seres humanos, como por exemplo a uveíte intermediária ou a doença de Behçet's (Gilger et al., 2000). O tratamento da uveíte é realizado de forma tópica, periocular, intraocular e sistêmica. Todavia, problemas são comumente relacionados à utilização de todas essas vias. Para superar esses efeitos colaterais, um número crescente de dispositivos de liberação lenta tem sido desenvolvidos, utilizando diferentes mecanismos de entrega e variedade de agentes farmacológicos.

Gilger e colaboradores (1999) desenvolveram um dispositivo intravítreo discoide para a liberação constante de ciclosporina (CSA) em casos de URE. Os níveis de proteína no humor vítreo e humor aquoso, infiltrado celular, o número total de linfócitos $T$ e demais parâmetros inflamatórios foram significativamente menores nos olhos que continham os implantes de CsA, em comparação com os olhos que continham placebo. Os implantes de CsA não foram capazes de eliminar completamente a doença, no entanto, a duração e gravidade da inflamação, o infiltrado celular, e a destruição tecidual foram menores nos olhos implantados com os dispositivos de CsA. Esse estudo se focou em demonstrara eficácia da entrega da molécula de CsA através de um dispositivo intraocular para o tratamento da inflamação imunomediada em um olho equino. Porém, a flexibilidade do dispositivo para a entrega de moléculas de fármacos e sua eficácia em outros modelos animais e em um modelo humano pode ser estudada e parece ser promissora. 
Implantes de PLGA contendo dexametasona e ciclosporina nas concentrações de $25 \%$ foram utilizados para o tratamento de URE no olho esquerdo de um animal da espécie eqüina com 20 anos de idade. Os implantes foram confeccionados pela equipe de farmacologia da Universidade Federal de Minas Gerais (UFMG) (Fialho e Silva Cunha, 2005; Fialho et al., 2006). Brevemente, os implantes são preparados pelo método de moldagem a quente, utilizando o polímero PLGA 50:50 da seguinte forma: primeiramente, o polímero e o fármaco foram dissolvidos em uma mistura de acetonitrila e água destilada. A solução resultante foi liofilizada (Liofilizador E-C MODULYO, E-C Apparatus Inc., EUA) para a obtenção de uma mistura homogênea. Esta mistura foi então utilizada para o preparo dos implantes. Na técnica de moldagem a quente, a mistura de pós previamente obtida foi moldada em bastões utilizando uma placa de teflon aquecida na temperatura de 100 a $120^{\circ} \mathrm{C}$. O peso médio do implante moldado foi de $4,7 \pm 0,3 \mathrm{mg}$, o diâmetro médio foi de $1,0 \pm 0,1 \mathrm{~mm}$ e o comprimento médio de $4,0 \pm 0,1 \mathrm{~mm}$. Os sistemas preparados por moldagem a quente apresentam uma superfície mais lisa com pouca evidência de poros e com aparência mais homogênea.

O animal foi avaliado pela equipe de oftalmologia veterinária da Universidade Federal do Paraná (UFPR). O olho esquerdo apresentava intenso edema corneano, neovascularização corneana, e eritema conjuntival (Figura 1). A miose era de difícil visualização, devido ao edema. Em adição, blefaroespasmo e fotofobia eram perceptíveis.

A pressão intraocular (PIO) era de $5 \mathrm{mmHg}$. O olho direito apresentava-se em phthisis bulbi, portanto não foi utilizado nesse estudo. Os implantes foram inseridos no vítreo do animal sob anestesia geral, na quantidade de dois implantes de cada fármaco. $\mathrm{O}$ animal foi reavaliado diariamente, sendo que no sexto dia pós-operatório verificou-se melhora visual significativa, com redução do edema e neovascularização corneana (Figura 2). A PIO nesse momento estava em $8 \mathrm{mmHg}$. 


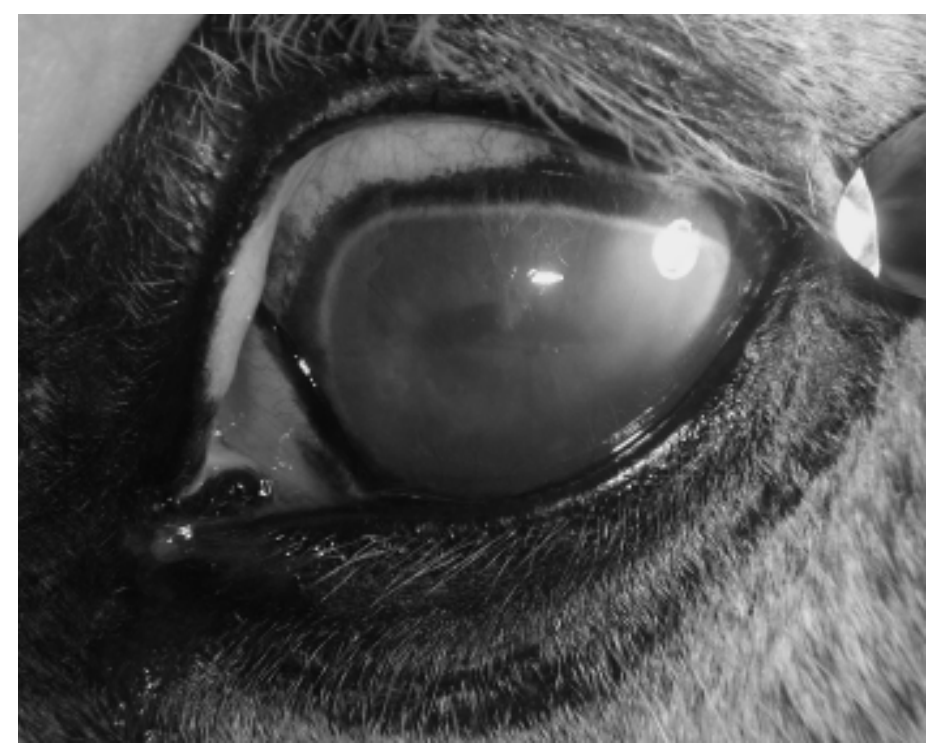

Figura 1 - Equino com URE antes da colocação de implantes intravítreos. Observe a intensa miose, sinéquia anterior e edema corneano, sinais clássicos de URE.

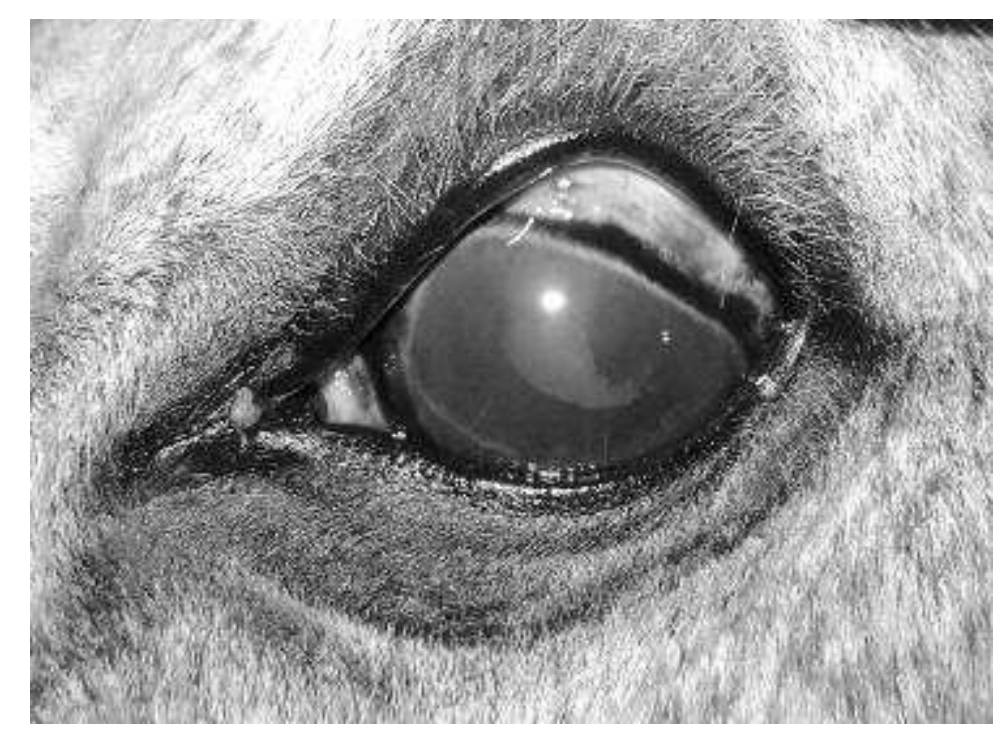

Figura 2 - Mesmo olho da figura 1, seis dias após a colocação dos implantes, demonstrando melhora significativa. Note a ausência de miose e diminuição do edema corneano. A sinéquia anterior persiste e fica mais visível.

No décimo quarto dia pós-operatório o animal voltou a apresentar sinais de URE, com piora do quadro. O proprietário optou pela eutanásia, não sendo 
possível a tentativa de reajuste e nova colocação dos implantes. Todavia, tratou-se de caso interessante para avaliar a eficácia desta forma de tratamento oftálmico. Obviamente alguns ajustes e mais pesquisas deverão ser realizados principalmente com relação à concentração, tempo de liberação do fármaco, confecção dos implantes e meios de implantação no olho do animal.

Como exemplo de implantes sólidos citados anteriormente, existe o Retisert ${ }^{\circledR}$ (Bausch \& Lomb, Rochester, NY, EUA), um implante contendo um orifício de álcool polivinil e uma cápsula de silicone que libera o corticosteróide fluocinolona em uma taxa de 0,4 a 0,6 $\mu \mathrm{g}$ por dia até aproximadamente 30 meses. Durante o estudo clínico aprovado pelo FDA, a recorrência de uveíte diminuiu de $54 \%$ para $7 \%$ após a implantação do Retisert $\Re$, aumentando ou estabilizando a acuidade visual em $80 \%$ dos pacientes. Efeitos oculares adversos incluem aumento da pressão intraocular (PIO) e formação de catarata (Haghjou et al., 2011).

Tendo como objetivo diminuir a quantidade variável de iridociclite pós operatória e o desconforto dos pacientes que passam por cirurgias intraoculares, a Oculex Pharmaceuticals Incorporated (Sunnyvale, California, EUA) desenvolveu e avaliou a eficácia de um implante intraocular de dexametasona (Surodex $($ ) para o tratamento de inflamações após cirurgias de catarata (Chang e Wong, 1999). Os pacientes que receberam o implante Surodex ${ }^{\circledR}$ tiveram menos eritema conjuntival, edema corneano, desconforto, dor ou fotofobia comparado com os pacientes do grupo controle. Ainda, os pacientes do grupo controle necessitaram medicação anti-inflamatória adicional mais frequentemente e com maior frequência de aplicação do que os pacientes do grupo Surodex $®$.

Como o sistema de liberação de fármacos na forma de implante deve ser biocompatível com o organismo, os componentes nele presentes devem ser não carcinogênicos, hipoalergênicos, mecanicamente estáveis e não 
causadores de resposta inflamatória no local de aplicação. Acrescenta-se, ainda, que as características químicas e físicas do material não devem ser modificadas pelo tecido local (Fialho et al., 2003). Sendo assim, pesquisas relacionadas à utilização dos polímeros biodegradáveis mostraram que eles proporcionaram boas propriedades mecânicas, baixa capacidade alergênica, baixa toxicidade, excelente biocompatibilidade e uma cinética previsível de biodegradação, despertando a atenção de vários pesquisadores quanto a suas possíveis aplicações em tecnologia farmacêutica.

\section{CONCLUSÃO}

Levando-se em consideração a realidade da medicina veterinária, onde várias espécies de animais são acometidas diariamente por doenças oculares crônicas, e necessitamos muitas vezes da adesão do proprietário ao tratamento, é possível afirmar que, reduzindo ou eliminando a responsabilidade e os encargos da administração múltipla de medicações muitas vezes de alto custo, obteremos maiores sucessos no tratamento destas doenças. Estes novos sistemas de liberação tem como objetivo melhorar o tratamento por meio da manutenção da concentração e do aumento da liberação do fármaco no local de aplicação e da redução da toxicidade intraocular. Dessa forma, a utilização de implantes intraoculares deve ser vista como uma forma inovadora de liberação de fármacos, que pretende revolucionar a terapêutica ocular nos âmbitos da medicina humana e animal, e devem ser considerados nos casos em que os efeitos secundários da terapia sistêmica ou tópica recorrente são mais frequentes ou graves do que os efeitos relacionados á colocação dos implantes. 


\section{REFERÊNCIAS}

AVITABILE, T.; MARANO, F.; CASTIGLIONE, F.; BUCOLO, C.; CRO, M.; AMBROSIO, L.; FERRAUTO, C.; REIBALDI, A. Biocompatibility and biodegradation of intravitreal hyaluronan implants in rabbits. Biomaterials, Guilford, v. 22, n. 3, p. 195-200, feb. 2001.

BEHAR-COHEN, F. Systèmes de délivrance des médicaments pour le segment antérieur: bases fondamentales et applications cliniques. Journal français d'ophtalmologie, Paris, v. 25, n. 5, p. 537-544, mai. 2002.

BISPO, V.M. Estudo do efeito da reticulação por genipin em suportes biocompatíveis de quitosana-PVA. 2009. Belo Horizonte, 206f. Dissertação (Doutorado em Engenharia Metalúrgica e de Minas) - Curso de Pós Graduação em Engenharia Metalúrgica e de Minas, Universidade Federal de Minas Gerais.

CHANG, D.F.; WONG, W. Two clinical trials of an intraocular steroid delivery system for cataract surgery. Transactions of the American Ophthalmological Society, v. 97: p. 261279, 1999.

CHOONARA, Y.E.; PILLAY, V.; CARMICHAEL, T.R.; MEYER, L.C.R.; TOIT L.C.D.; NAYLOR, S.; WANBLAD, C. In Vivo evaluation of a biodegradable donut-shaped minitablet for prolonged posterior segment drug delivery in the rabbit eye model. Journal of Pharmaceutical Sciences, v.100, n. 5, p 1819-1832, mai 2011.

DASH, A. K.; CUDWORTH II, G. C. Therapeutic applications of implantable drug delivery systems. Journal of Pharmacological and Toxicological Methods, NewYork, v. 40, n. 1, p. $1-12$, jul. 1998.

DEEG, C.A.; KASPERS, B.; GERHARDS, H.; THURAU, B.W.; WILDNER, G. Immune responses to retinal autoantigens and peptides in equine recurrent uveitis. Investigative Ophthalmology \& Visual Science, v.42, n. 2, p. 393-398, feb 2001.

FIALHO, S.L.; CUNHA JÚNIOR A.S. Sistemas de transporte de drogas para o segmento posterior do olho: bases fundamentais e aplicações. Arquivo Brasileiro de Oftalmologia, v.70, n.1, p. 173-179, 2007.

FIALHO, S. L.; REGO, M. G. B.; CARDILLO, J. A.; SIQUEIRA, R. C.; JORGE, R.; SILVA-CUNHA, A. Implantes biodegradáveis destinados à administração intraocular. Arquivos Brasileiros de Oftalmologia, São Paulo, v. 66, n.6, p.891-896, dez. 2003.

FIALHO, S.L.; REGO, M.B.; SIQUEIRA, S.C.; JORGE, R.; HADDAD, A.; RODRIGUES JR, A.L.; MAIA-FILHO, A.; SILVA-CUNHA, A. Safety and pharmacokinetics of an intravitreal biodegradable implant of dexamethasone acetate in rabbit eyes. Current Eye Research, v. 31 , p. $1-10,2006$.

FIALHO, S. L.; SILVA-CUNHA, A. Manufacturing techniques of biodegradable implants intended for intraocular application. Drug delivery, Orlando, v. 12, n. 2, p. 109-116, mar/apr. 2005.

GILGER, B.C.; MALOK, E.; CUTTER, K.V.; STEWART, T.; HOROHOV, D.W.; ALLEN, J.B. Characterization of T-lymphocytes in the anterior uvea of eyes with chronic equine recurrent uveitis. Veterinary Immunology and Immunopathology, v. 71, p. 17-28, 1999.

GILGER, B.C.; MALOK, E.; STEWART, T.; HOROHOV, D.; ASHTON, P.; SMITH, T.; JAFFE, G.J.; ALLEN, J.B. Effect of an intravitreal cyclosporine implant on experimental uveitis in horses.

Veterinary Immunology and Immunopathology, v.76, p. 239-255, 2000. 
HAGHJOU, N.; SOHEILIAN M.; ABDEKHODAIE M.J. Sustained release intraocular drug delivery devices for treatment of uveitis. Journal Ophthalmic Visual Research, v.6, n.4, p. 317-329, 2011.

HASHIZOE, M.; OGURA, Y.; KIMURA, H.; MORITERA, T.; HONDA, Y.; KYO, M.; HYON, S-H.; IKADA, Y. Scleral plug of biodegradable polymers for controlled drug release in the vitreous. Archives of Ophthalmology, Chicago, v. 112, n. 10, p. 1380-1384, oct. 1994.

JAIN, R.; SHAH, N. H.; MALICK, A. W.; RHODES, C. T. Controlled drug delivery by biodegradable poly (ester) devices: different preparative approaches. Drug Development and Industrial Pharmacy, New York, v. 24, n. 8, p. 703-727, aug. 1998.

KELLER, R.L.; HENDRIX, D.V.H. New surgical therapies for the treatment of equine recurrent uveitis. Clinical Techniques in Equine Practice, p. 81-86, 2005.

KIMURA, H.; OGURA, Y. Biodegradable polymers for ocular drug delivery. Ophthalmologica, Basel, v. 215, n. 3, p. 143-155, may./jun. 2001.

OKABE, J.; KIMURA H.; KUNOU N.; OKABE K.; KATO A.; OGURA Y. Biodegradable intrascleral implant for sustained intraocular delivery of betamethasone phosphate. Investigative

Ophthalmology and Visual Science, Rockville, v. 44, n. 2, p. 740-744, feb. 2003.

RUBSAMEN, P. E.; DAVIS, P. A.; HERNANDEZ, E.; O'GRADY, G. E.; COUSINS, S. W. Prevention of experimental proliferative vitreoretinopathy with a biodegradable intravitreal implant for the sustained release of fluorouracil. Archives of Ophthalmology, Chicago, v. 112, n. 3, p. 407413, mar. 1994.

SAKURAI, E.; NOZAKI, M.; OKABE, K.; KUNOU, N.; KIMURA, H.; OGURA Y. Scleral plug of biodegradable polymers containing tacrolimus (FK506) for experimental uveitis. Investigative Ophthalmology and Visual Science, Rockville, v. 44, n. 11, p. 4845-4852, nov. 2003.

SALIBA, J.B.; FARACO A.G.; YOSHIDA M.I.; VASCONCELOS W.L.; SILVA-CUNHA, A.; MANSUR, H.S. Development and characterization of na intraocular biodegradable polymer system containing cyclosporine-A for the treatment of posterior uveitis. Materials Research, v.11, n.2, p. 207-211, 2008.

THENG, J. T.; SENG-EI, T.; ZHOU, L.; LAM, K. W.; CHEE, S. P.; TAN, D. Pharmacokinetic and toxicity study of an intraocular cyclosporine dds in the anterior segment of rabbit eyes.

Investigative Ophthalmology and Visual Science, Rockville, v. 44, n. 11, p. 4895-4899, nov. 2003. 